\title{
A Prospective Study among Patients with Chronic Headache and Relation with Computed Tomography Findings attending a Tertiary Care Hospital of South India
}

\author{
Prashanth Thimme Gowdaㄹ, Pannem Ramesh Babu \\ ${ }^{1}$ Associate Professor, Department of Radiodiagnosis, ${ }^{2}$ Associate Professor, Department of General Medicine, Narayana \\ Medical College Chinthareddyaplem, Nellore, Andhra Pradesh, India
}

Corresponding author: Dr. Pannem Ramesh Babu, Associate Professor, Department of General Medicine, Narayana Medical College Chinthareddyaplem, Nellore, Andhra Pradesh, India

DOI: http://dx.doi.org/10.21276/ijcmsr.2018.3.4.18

How to cite this article: Prashanth Thimme Gowda, Pannem Ramesh Babu. A prospective study among patients with chronic headache and relation with computed tomography findings attending a tertiary care hospital of South India. International Journal of Contemporary Medicine Surgery and Radiology. 2018;3(4):D78-D81.

\section{A B S T R A C T}

Introduction: Headache is one of the common clinical illnesses with a lot of discomfort encountered in clinical practice. Population based studies in US have reported that headache causes a loss of 150 million work days per year. The increasing availability of computed tomography and at a reasonable price has fuelled enthusiasm for its role in ruling out brain tumours and any secondary neurological conditions. The primary aim of the study is to assess the utility of computed tomography in identifying the causes of chronic headache

Material and methods: A prospective descriptive study was conducted for two years on all patients attending the general medicine OPD with chronic headache. All the cases who fulfilled the inclusion criteria were subjected to computed tomography by 128 slice MDCT (Toshiba Aquallion) scanner and detailed clinical examination. IV contrast was used on selected patients depending on the necessity. All the findings were noted and analyzed using statistical package for software version 19. Chicago. Significance level was fixed at $p<0.05$.

Results: 250 cases with 164 (65.6\%) males and 86 (34.4\%) females were included. 41-55 years was the most common age group with mean age of 34 years. Abnormal CT findings were observed in 31 cases, with 21 male and 10 females. $49.6 \%$ presented with 3-6 months duration of headache. Para nasal sinusitis (predominantly maxillary with associated frontal, ethmoid sinuses), space occupying lesions, Infracts (Ring enhancing lesions) were the most common findings (6 cases each of $31,19.35 \%$ ) in our study.

Discussion: To conclude CT is a useful screening modality in evaluating cases of headache either to identify or rule out structural abnormality. Diagnostic yield from CT is better in patients with severe headache or headache of sudden onset or associated with blurring of vision or nausea and vomiting or any neurological deficits.

Key words: Computed Tomography, Headache, Paranasal Sinusitis, Infracts

\section{INTRODUCTION}

Headache is one of the common clinical illnesses with a lot of discomfort encountered in clinical practice. This condition usually affects the daily activity of the patient as well as mental health status of the patients. $90 \%$ of individuals have at least one episode of headache during their lifetime and severe headache is reportedly to occur in $40 \%$ of population. Hokkanen and Pirskanen reported that the prevalence rate of chronic headache may be as high as $95 \%$ among women and $69 \%$ among men. ${ }^{1}$ Population based studies in US have reported that headache causes a loss of 150 million work days per year. This may be acute or chronic with variable underlying causes. However the primary causes are the majority, with tension headache being the common cause and only less than $10 \%$ will have secondary cause which may be due to underlying pathology (Eg: Aneurysm or SOL) etc. Numerous studies have helped to identify the clinical symptoms and signs, those when associated with headache appear to be useful predictors of positive imaging and evaluation and justify neuroimaging.

The relative rarity of secondary headaches compared with large number of cases with primary headache raises the concern of Neuroimaging studies like computed tomography to exclude underlying causes of headache. ${ }^{2}$ Neuroimaging relieves the patient's anxiety and apprehension of possessing a secondary pathological condition which improves the patient medical care and satisfaction. Other major reason for obtaining Neuroimaging is at the request of the family or the tensed patient. The increasing availability of computed tomography and at a reasonable price has fuelled enthusiasm for its role in ruling out brain tumours and any secondary neurological conditions. Numerous studies have helped to identify the clinical symptoms and signs, those when 
associated with headache appear to be useful predictors of positive imaging evaluation and justifying Neuroimaging in cases of chronic headache. ${ }^{3}$ The primary aim of the study was to assess the utility of computed tomography in identifying the causes of chronic headache.

\section{MATERIAL AND METHODS}

A prospective study was conducted at a tertiary care hospital among the patients attending the department of general medicine with chief complaint of headache with or without other associated symptoms for a period of two years from January 2016 to December 2017. The study design was approved by the institutional ethical committee and the study protocol was followed as per the guidelines of the committee. All the cases of headache attending to the OPD of general medicine department were examined by a senior physician and the socio demographic data, history of clinical signs and symptoms (duration, localization, medication history etc) were noted and entered by a senior physician into separate predesigned questionnaire sheet. The study details were explained to the cases in the study and written informed consent was obtained from all the cases in the study. All the cases consented for the study were sent to the department of radio diagnosis and evaluated for computed tomography of the brain. The procedure was clearly explained to all the cases in the study. The study was done using a 128 slice MDCT (Toshiba Aquallion) scanner. CT of the brain was performed with patient in supine position and the plane of the scan taken parallel to orbito- meatal plane at $10-25^{0}$ to Reid's line. Plain (without contrast) and with contrast enhanced CT scans were performed. The findings of the scans were noted in the predesigned questionnaire sheet. Intravenous iodinated contrast media was used for contrast study of brain in selected patients with an average dose of $25 \mathrm{cc}$.

Inclusion criteria: Patients $>10$ years of age, as history of headache is difficult to elicit from children.

Cases who consented for the study.

Exclusion Criteria: Cases with known intracranial abnormality eg: Aneurysm, VP shunt etc.

Headache due to ophthalmic causes.

Headache due to trauma.

Pregnant females.

\section{STATISTICAL ANALYSIS}

The data was tabulated and analyzed using statistical package for software. (SPSS version 19. Chicago USA) data was compared by applying Chi-Square test to test level of significance. Qualitative variables were compared using proportions. Significance level was fixed at $\mathrm{p}<0.05$.

\section{RESULTS}

A prospective study was conducted for a period of two years and 250 cases who fulfilled the inclusion criteria were enrolled in the study. The selected patients were evaluated by performing Computed tomography of the brain. Plain CT of the brain was performed in 198 cases and 52 cases with Intravenous contrast enhancement. Of the 250 cases enrolled $164(65.6 \%)$ were males and 86 (34.4\%) were females. Among the 164 males, plain CT was performed in 145 cases and CT with contrast in 19 cases. Among the 86 female cases in the study, Plain CT was performed in 54 cases and 33 with contrast. A total of 31 cases of 250 reported abnormal CT findings, with 20 cases (11.24\%) on plain CT and 11

\begin{tabular}{|c|c|c|c|c|c|c|c|}
\hline Age group (In Years) & Male & Female & \multicolumn{2}{|c|}{ Total (No) (\%) } & Abnormal & Chi-Square & $P$ value \\
\hline $10-25$ & 11 & 5 & 16 & 6.4 & 2 & \multirow[t]{5}{*}{4.82} & \multirow{5}{*}{$\begin{array}{c}0.38 \\
\text { (Not } \\
\text { Significant) }\end{array}$} \\
\hline $26-40$ & 28 & 18 & 46 & 18.4 & 11 & & \\
\hline $41-55$ & 54 & 38 & 92 & 36.8 & 8 & & \\
\hline $56-70$ & 48 & 12 & 60 & 24 & 4 & & \\
\hline \multirow[t]{2}{*}{$>70$ years } & 23 & 13 & 36 & 14.4 & 6 & & \\
\hline & 164 & 86 & \multicolumn{2}{|c|}{250} & 31 & & \\
\hline
\end{tabular}

\begin{tabular}{|c|c|c|c|c|c|c|}
\hline S. No & Diagnosis & Male (No) & Female (No) & No of cases & $\begin{array}{l}\text { Chi-square } \\
\text { value }\end{array}$ & P value \\
\hline 1 & Para Nasal Sinusitis & 4 & 2 & $6(19.35)$ & \multirow[t]{8}{*}{6.42} & \multirow{8}{*}{$\begin{array}{c}0.04 \\
\text { (Significant) }\end{array}$} \\
\hline 2 & Bone related pathology & 2 & & $2(6.45)$ & & \\
\hline 3 & CSOM & 3 & 1 & $4(12.90)$ & & \\
\hline 4 & Cortical vein Thrombosis & 2 & & $2(6.45)$ & & \\
\hline 5 & Space occupying Lesions (SOL) & 3 & 3 & $6(19.35)$ & & \\
\hline 6 & Infarcts (Ring enhancing Lesions) & 3 & 3 & $6(19.35)$ & & \\
\hline 7 & Parenchymal, SAH \& SDH & 3 & 1 & $4(12.90)$ & & \\
\hline \multirow[t]{2}{*}{8} & Aneurysm & 1 & 0 & $1(3.23)$ & & \\
\hline & Total & 21 & 10 & 31 & & \\
\hline
\end{tabular}




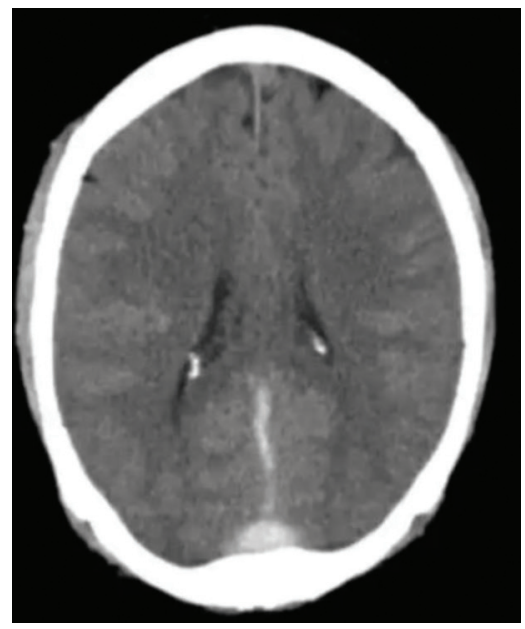

Figure-1: A case of Cortical venous thrombosis

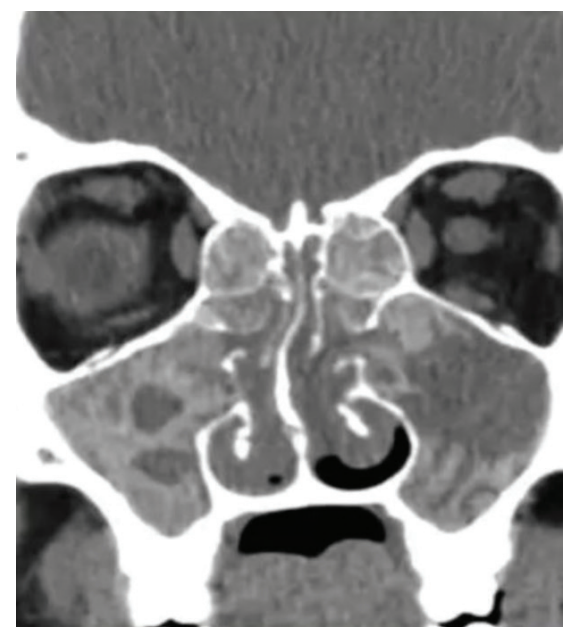

Figure-2: A case of Pansinusitis.

(26.83\%) on CT with contrast. Of the total 31 cases with abnormal CT findings, 21 (12.80\%) were males and 10 cases $(11.63 \%)$ were females. The most common age group in the study was between $41-55$ years with $36.8 \%$ followed in order by $26-40$ years $(18.4 \%),>70$ years $(14.4 \%)$ and least $6.4 \%$ in age group of 10-25 years. Abnormal CT findings were high (11 cases) in the age group of 26-40 years followed next in order by 8 cases in the age group of 41-55 years. However there was no significant association between age group and abnormal CT findings in the study. ( $\mathrm{P}$ vale $>0.05)$ [Table-1] The mean age of the cases in the study was 34 years.

With regarding to duration of headache, majority (49.6\%) presented with 3-6 months and least with $>6$ months $(8.4 \%)$. There was no significant association between duration of headache and abnormal CT findings in the study. ( $\mathrm{P}$ value $>0.05$ ) Other associated symptoms in the study were fever, Nausea and vomiting, blurring of vision, systemic hypertension, none of the symptoms had significant association with abnormal CT findings in the study.

Table-2 summarizes the frequency of distribution of cases according to the disease findings in the study. Para nasal sinusitis (predominantly maxillary with associated frontal, ethmoid sinuses), space occupying lesions, Infracts (Ring enhancing lesions) were the most common findings (6 cases each of $31,19.35 \%$ ) in our study with less common findings which include CSOM, parenchymal, sub-dural and subarachnoid haemorrhages (4 cases each, 12.9\%) Cortical vein thrombosis, bone related pathology (2 cases each, 6.45\%) and the aneurysm in one case in entire study (3.23\%). [Fig-1\&2] Significant association was observed between cases presented with headache and abnormal findings on computed tomography. ('p'value $<0.05$ )

\section{DISCUSSION}

Headache is one of the common ailments encountered in clinical practice. The International society for headache has classified it in to two types; primary without any organic cause and secondary with an underlying cause. ${ }^{4}$ Secondary headache require Neuroimaging for diagnosis and clinical assessment alone is not sufficient. Various studies have been done to evaluate the ability of Neuroimaging in evaluation of chronic or recurrent headaches without neurological abnormality. In the present study there was a male predominance, however various studies reports in general headache is more common in females than males. However tension headache is more common among females and cluster headache is more common among males. The most common age group in the study was $41-55$ years with range from 18-72 years. The median age of the study population was 34 years. Similar results were observed in the study of Kahn CE Jr et al who reported that abnormalities on CT were associated with increased age..$^{5}$ In the present study, a total of 31 cases exhibited abnormal findings on computed tomography, 21 (12.80\%) were males and 10 cases $(11.63 \%)$ were females. There was no significant difference in gender with relating to cause of headache in imaging. Few studies reported that abnormal findings were significantly higher among females than males which are contrary to the finding of our study. This difference is attributed to the presence of more female cases in the study and different parameters of consideration in the study, eg: Ophthalmic causes, migraine etc. However study conducted at Nepal, demonstrated that $64.3 \%$ of cases had normal CT scans and $35.7 \%$ had abnormal findings. ${ }^{6}$ According to a meta-analysis significant clinical abnormalities detected in CT scans in patients with unspecified headache ranged from $0.0 \%$ to $6.7 \%$ in ten studies. $^{7}$

In the present study, Maximum cases presented with duration of headache between 3-6 months, and there was no significant association between duration of headache and abnormal findings. Findings of our study were on par with findings of Evans RW who reported no significance between duration of headache and abnormal CT findings, but contrary to the reports of Alders EE et al who reported significant association between duration of headache, blurring of vision and vomiting in his study. ${ }^{8,9}$ In the present study no significant association was observed between any signs and abnormal findings on CT.

In the present study, 52 cases were administered contrast and $26.83 \%$ reported abnormal findings. Our study highlights the importance of Contrast administered CT in evaluation of headache than Plain CT. There was a statistically significant association observed between contrast administered CT and abnormal CT findings in our study which is similar to the 
findings of Medina LS but contrary with the results of Gaini SM who reported no significant association between Plain and contrast enhanced CT in detection of abnormalities on CT. ${ }^{10,11}$

Headache without any associated symptoms or neurological deficit was the main reason for referral of patients for neuroimaging followed by headache with vomiting. These observations were similar to the findings of Sherf $M$ et al, to evaluate the indications and results for brain CT by primary care physicians. ${ }^{12}$ Paranasal sinusitis was one of the common abnormal finding seen in 6 cases which is similar to the study reports of Subedee A et al but contrary to the reports of Evans $\mathrm{R}$ who reported SOL of brain as the most common cranial abnormal finding on $\mathrm{CT}$ in his study. ${ }^{13,14}$ No abnormality was detected in cases with cluster headache. The yield from imaging in cases of primary headache is low which is reported by many studies universally. However cases of migraine in our study were low and the rate of detection of any abnormality in these cases were low in our study. Similar findings were reported by Valade et al in their study. ${ }^{15}$ Most of the cases of sinusitis had focal headache in our study. Among the 6 cases of infarcts in our study, 2 cases had vision abnormality which suggests a high probability of abnormal $\mathrm{CT}$ finding in cases with headache and vision abnormality. Headache with nausea and vomiting had a less probability of abnormal imaging in our study which is similar to the findings of Mitchell et al in his study. ${ }^{16}$

Similar to many studies earlier, many cases were apprehensive with fear of having abnormality in brain. In these patients CT helps us to rule out any possibility of organic cause for headache there by reducing patient apprehension. Perceived disadvantages of CT as an imaging modality will be in the evaluation of sellar and parasellar causes of headache, posterior fossa lesions, characterization of lesions and radiation exposure.

\section{CONCLUSION}

To conclude CT is a useful screening modality in evaluating cases of headache either to identify or rule out structural abnormality. Diagnostic yield from CT is better in patients with severe headache or headache of sudden onset or associated with blurring of vision or nausea and vomiting or any neurological deficits.

\section{REFERENCES}

1. Pakarinen S: Incidence, aetiology and prognosis of primary subarachnoid haemorrhage. Acta Neurol Scand 1967; 43 (suppl 29):1-128.

2. Frishberg BM, et al. Evidence-based guidelines in the primary care setting: neuroimaging in patients with non-acute headache. Available from: URL: http://www. aan.com/public/practice guidelines (2000).

3. Larson, E., Omenn, G. and Lewis, H. Diagnostic evaluation of headache. Impact of computerized tomography and cost-effectiveness. JAMA 1980;243(3): 359-362.

4. Headache Classification Subcommittee of the International Headache Society. The International Classification of Headache Disorders. Cephalalgia
2004;24(1):160.

5. Kahn CE Jr, Sanders GD, Lyons EA et al.Computed tomography for non-traumatic headache: current utilization and costeffectiveness. Can Assoc Radiol J 1993; 44(3):189-193.

6. Nepal P, Shrestha A, Ghimire N. Evaluation of CT Scan Findings in Patients Presenting with Headache. Journal of Chitwan Medical College 2014;3(4): 9-12.

7. Frishberg BM, Rosenberg JH, Matchar DB, et al. Evidence-based guideline in the primary care setting: neuroimaging in patients with nonacute headache. U.S. Headache Consortium.

8. Evans RW. Diagnostic testing for migraine and other primary headaches. Neurologic clinics 2009;27(2):393415.

9. Alders EE, Hentzen A, Tan CT. A community-based prevalence: study on headache in Malaysia. Headache: The Journal of Head and Face Pain 1996;36(6):379-84.

10. Medina LS, D'Souza B, Vasconcellos E. Adults and children with headache: evidence based diagnostic evaluation Neuroimaging Clin N Am 2003;13(2):22535 .

11. Gaini SM, Fiori L, Cesana C, Vergani F. The headache in the Emergency Department. Neurol Sci 2004;25 Suppl 3:S196-201.

12. Sherf $\mathrm{M}$ et al. Evalaute the indications and results of referrals of brain computed tomography (CT) by primary care physicians. Eur J Neurol 2003;33(3):182196.

13. Subedee A. Evaluation of chronic headache by computed tomography:A retrospective study. J Nobel Med Coll 2012;1(5):57-63.

14. Evans, R. Diagnostic testing for the evaluation of headaches. Neurol Clin 1996; 14(6): 1-26.

15. Valade, D., et al., Migraine diagnosis and management in general emergency departments in France. Cephalalgia 1999.31(4): p. 471-80.

16. Mitchell CS, Osborn RE, Grosskreutz SR. Computed tomography in headache patient: Is rotine evaluation really necessary? Headache 1993;33(2); 82-86.

Source of Support: Nil; Conflict of Interest: None

Submitted: 20-10-2018; Accepted: 22-11-2018; Published online: 28-11-2018 